\title{
Access to Healthcare among the Adult Population
}

\author{
Henry O’Lawrence ${ }^{1 *}$, Linda Martinez ${ }^{1}$ \\ ${ }^{1}$ Department of Healthcare Administration, College of Health and Human Services, California State University, Long \\ Beach, CA, USA
}

*Corresponding Author: henry.olawrence@csulb.edu

Citation: O'Lawrence, H. and Martinez, L. (2018). Access to Healthcare among the Adult Population. European Journal of Environment and Public Health, 2(2), 05. https:/ / doi.org/10.20897/ ejeph/90840

Published: September 28, 2018

\begin{abstract}
This study investigates the level of access to healthcare among the elderly population in California. A total of 21,055 members of the adult population, ages from 18 to 65 participated in this survey. The majority of participants, about $61 \%$, reported they are generally in good or a very good health condition compared to $39 \%$ who reported that they are in fair or very poor condition. The overall purpose of this study was to evaluate access to healthcare services among the adult population in the context of insurance status. Lack of access to healthcare services among the population is a societal and moral concern nationwide and there is a need to identify common characteristics among the targeted demographic and provide an opportunity for prevention and action.
\end{abstract}

Keywords: health care reform, elderly population, access, health care services

\section{INTRODUCTION}

Access to quality health care is a major concern in the U.S., even with the implementation of the Affordable Care Act (ACA) of 2010 and the key features of the law that include rights and protection of healthcare consumers, insurance choice and insurance costs (Niles, 2018). With the broad parameters of the ACA, the way to move ahead with the reforms still remains elusive as the landscape for health care continues to shift and unfortunately enough, people are increasingly becoming unhealthy even though the U.S. is spending more on health care per capital than any other nation (Hammaker and Knadig, 2017).

The major reforms that were implemented in 2010 included provisions that eliminated lifetime and unreasonable annual caps or limits on healthcare reimbursement with annual limitations prohibited in 2014. The provision also provided assistance for the uninsured with preexisting conditions and prohibited denial of insurance coverage for preexisting conditions for children; and developing a temporary national high-risk pool for health insurance for individuals with preexisting conditions who have no insurance. Significantly, the reforms that were implemented in 2010 also provided data for consumers online to access reports about healthcare insurance, create a reinsurance program for retirees who are not yet eligible for Medicare and extended dependent coverage up to 26 years of age (Niles, 2018).

There are many factors leading to lack of access to healthcare and most especially, among individuals, families and undocumented immigrants with low socioeconomic status. These groups will either forgo healthcare services, seek care from a community health clinic or use the emergency department for primary or non-emergent services. The weight of these groups creates a cost burden that could threaten the sustainability of government funded organizations (Bisgaier and Rhodes, 2011).

A major challenge facing the nation's population is the level of inequality in the health care access and it is determined that lack of access to affordable health insurance is one of the shortcomings in the U.S. health care 
system compared to other similar higher income, developed countries. Among other major factors like life expectancy, lack of universal access to medical coverage is the largest major "contribution to the underperformance of the U.S. health care systems in terms of broad population health measures." There are 33 million uninsured people or 1 in 10 in the United States with no health insurance, leading the uninsured to risk serious illness or death by delaying necessary care. Lack of health insurance contributes to more than 45,000 unnecessary deaths every year (Hammaker and Knadig, 2017, p. 94).

Bisgaier and Rhodes (2011) also identified socioeconomic status segments (SES) that contained numerous factors such as, social class (i.e., individual, household and neighborhood), race and ethnicity, gender, educational level, income and financial constraints. Previous studies also documented that ethnicity and minorities' patients in this country are disproportionately affected by many health care conditions that affect their health in comparison to white patients. Significantly, lack of access to health care due to lack of health insurance are cited for health disparities. In most cases, lack of health insurance coverage resulted in ethnic minorities refraining from healthcare services, resulting in health deterioration and consequently requiring more extensive treatment; whereas, if the condition had been detected earlier, it could have be eradicated or maintained. Therefore, racial and ethnic groups continue to experience an impediment to the access of healthcare, especially undocumented immigrants who have low incomes (Chen, 2010).

Lewis et al. (2012) identified two categories of vulnerable populations as, clinically at risk (i.e., high risk patients who have chronic conditions or are susceptible to poor health) and socially disadvantaged (i.e., low socioeconomic factors, destitute living situation, minorities and minimal social support), with the most vulnerable population being the individuals that fall within both realms.

The health care costs of uninsured in terms of uncompensated care are helping drive up overall costs and the spillover effect of not being insured is more disturbing, as the uninsured with chronic illnesses are less likely to receive appropriate care to manage their health conditions and have consistently worse clinical outcomes than do comparable insured patients. Most significantly, half of the uninsured young adults experience difficulties paying off their medical bills and 1 in 5 among them were uninsured before 2010; today, almost 1.8 million young adults remain uninsured and have the lowest rate of access to employer-based insurance (Hammaker and Knadig, 2017).

\section{PURPOSE OF THE STUDY}

Prior research has looked into access to healthcare services among the poor, uninsured and minorities without much on adult population and barriers in accessing health care services (Dia et al., 2011; Johnson and Bao, 2011). Therefore, the purpose of this study was to examine accessibility to healthcare services in non-elderly adults, while identifying distinctions between the population and what characteristics contribute to the deficiency in access to healthcare services. While prior research has looked at access to healthcare services among the poor, uninsured, and minorities, there is a scarcity of research looking specifically at non-elderly adults and barriers in accessing healthcare services. The enactment of the ACA expanded coverage to additional recipients, such as the poor and non-elderly adults. However, there is still a concern that the neediest or most vulnerable non-elderly adults will fall between the gaps. Individuals who are socially disadvantaged and who are considered clinically vulnerable are continuously faced with barriers when it comes to accessing healthcare services (Lewis et al., 2012).

\section{METHODOLOGY}

A secondary data set from California Health Interview Survey (CHIS) adult questionnaire, covering the 2016 calendar year was used in this study. For point of clarification, non-adult populations were considered ages between 18-64 years old.

The CHIS adult questionnaire for the year 2016 provided insight to access to care by addressing the following questions: Does the lack of health insurance and lack of available resources result in a higher likelihood that nonelderly adults will forgo healthcare until their health condition worsens and what are the disparities found among non-elderly adults and access to care?

The 2016 California Health Interview Survey (CHIS) consists of telephone interviews, and data compilation; data release occurred biennially. To ensure adequate representation of California's diverse population, the questionnaire was given in five languages (English, Spanish, Mandarin and Cantonese Chinese dialects, Vietnamese, and Korean. CHIS offers high quality data, which correctly encompasses both the diversity of California and the communication methods of society today. 


\section{RESULTS}

The Statistical Package for Social Sciences (SPSS) was utilized for these results. The CHIS questionnaire, included: self-reported racial / ethnic groups, educational attainment, poverty levels, source of healthcare, health condition, delay in medical care and insurance status. In reference to common daily symptoms of High-Blood Pressure (HBP) they may have, only 25\% Californians indicated that they have issues with high-blood pressure compared to $60 \%$ that do not have the symptoms. About $30 \%$ of those with HBP are taking regular medication and only $9 \%$ are not taking drugs to control their high-blood pressure.

One of the questions asked if a doctor ever told them they have high blood pressure, and about $38.7 \%$ said that doctor told them they have high blood pressure and $60.2 \%$ said never. About $15 \%$ of the participants indicated that their doctor told them that they have borderline diabetes and $83 \%$ said that their doctor has not told them anything. The findings also suggested that Californians are very active and in good health generally. About $96 \%$ of the participants indicated that they walk at least 10 minutes or more for leisure every week.

For general health condition, $16.1 \%$ indicated that they are in excellent health condition, $29 \%$ reported that they are in a very good condition, $31.1 \%$ in good condition, and $17.4 \%$ in fair condition; and, only $6 \%$ said that they are in poor condition. For how often they eat fresh fruits and vegetables, significantly $75.7 \%$ of people indicated that they always consume fresh fruits and vegetables. Table 1 below shows the responded affordability to fresh fruits and vegetables.

Table 1. Participants responses to fruit and vegetables affordability

\begin{tabular}{lcc}
\hline Questions & Total Number & Total \% \\
\hline Never & 851 & 4.0 \\
\hline Sometimes & 1807 & 8.6 \\
\hline Usually & 2297 & 10.9 \\
\hline Always & 15948 & 75.7 \\
\hline Doesn't eat/shop for fruits and vegetables & 152 & .7 \\
\hline Total & 21055 & 100.0 \\
\hline
\end{tabular}

Also, a majority of participants regularly see their doctors at least once a year. However, it is significantly higher among those who do not have any doctor visits for the past year compared to those who had doctor visits at least one time in the past year. Table 2 bellow is the breakdown of doctor visits within a year.

Table 2. Responded number of doctor visits in the past year

\begin{tabular}{lcc}
\hline How many times & Total number & Total $\%$ \\
\hline 0 Time & 3177 & 15.1 \\
\hline 1 Time & 3821 & 18.1 \\
\hline 2 Times & 3588 & 17.0 \\
\hline 3 Times & 2561 & 12.2 \\
\hline 4 Times & 2093 & 9.9 \\
\hline 5 Times & 1125 & 5.3 \\
\hline 6 Times & 1223 & 5.8 \\
\hline $7-8$ Times & 724 & 3.4 \\
\hline 9-12 Times & 1595 & 7.6 \\
\hline $13-14$ Times & 736 & 3.5 \\
\hline 25+ Times & 412 & 2.0 \\
\hline Total & 21055 & 100.0 \\
\hline
\end{tabular}

$4 \%$ who did not do so and about $20 \%$ who indicated that the question is not applicable. About $86 \%$ indicated that they were covered for prescription drugs and only $8.3 \%$ said no and 5.7\% did not answer the question or indicated that the question is inapplicable.

\section{CONCLUSION}

The type of current health coverage sources was also looked into and it was found that a majority of the adults have coverage through their employers. Those who have employment-based insurance coverage amount to $30 \%$, Medicaid $19.6 \%$, privately purchased $5.5 \%$, Medicare $1 \%$, others $.9 \%$ and uninsured $6 \%$. The rest of $36.9 \%$ did not respond to the question or skipped the question. Findings showed that there is a relationship between access to healthcare services, healthcare coverage and delay in medical care. There is also a relationship between having a usual source of care to obtain healthcare services, healthcare coverage, delays in obtaining medical care and health condition. 
There was however a moderate number of people who reported having no usual source of care, both uninsured and insured. With the most common reason for lack of healthcare coverage, being that insurance was too expensive and therefore they could not afford purchasing healthcare insurance. The data also reveals that the majority reported no delay in medical care. However, people who reported having a reliable source of care are also experiencing delay in medical care and the uninsured managed to obtain care and experienced no delay in medical care. The debate regarding the quality of the U.S. healthcare system relative to other developed countries continues to be a major concern to many tax payers; whereas, many physicians for a National Health programs claimed that a free market solution to healthcare provides lower quality of care with higher mortality rates than the publicly funded systems.

\section{REFERENCES}

Bisgaier, J. and Rhodes, K. V. (2011). Cumulative adverse financial circumstances: Associations with patient health status and behaviors. Health \& Social Work, 36(2), 129-137. https:/ / doi.org/10.1093/hsw/36.2.129

California Health Interview Survey. (2017). CHHIS 2016 adult survey data file. UCLA center for health policy research. Los Angeles, CA.

Chen, L. (2010). Is there disparity in physician service use? A comparison of Hispanic and White Medicare beneficiaries. Health \& Social Work, 35(3), 181-190. https://doi.org/10.1093/hsw/35.3.181

Dias, S., Gama, A., Cortes, M. and deSousa, B. (2011). Healthcare-seeking patterns among immigrants in Portugal. Health and Social Care in the Community, 19(5), 514-521. https:// doi.org/10.1111/j.1365-2524.2011.00996.x

Hammaker, D. K. and Knadig, T. M. (2017). Health care ethics and the law. Jones \& Bartlett Learning, Burlington, MA.

Johnston, V. and Bao, Y. (2011). Race/ethnicity-related and payer-related disparities in the timeliness of emergency care in the U.S. emergency departments. Journal of Healthcare for the Poor and Underserved, 22(2), 606-620. https://doi.org/10.1353/hpu.2011.0050

Lewis, V. A., Larson, B. K., McClurg, A. B., Boswell, R. G. and Fisher, E. S. (2012). The promise and peril of accountable care for vulnerable populations: A Framework for overcoming obstacles. Health Affairs, 31(8), 1777-1785. https://doi.org/10.1377/hlthaff.2012.0490

Niles, N. C. (2018). Basics of the U.S. health care system. Third Edition. Jones \& Bartlett Learning, Burlington, MA. 